\title{
Numerical and Theoretical Study of the Crosstalk in Gain Clamped Semiconductor Optical Amplifiers
}

\author{
Jinying Sun, Geert Morthier, Member, IEEE, and Roel Baets, Member, IEEE
}

\begin{abstract}
A rate equation model of a gain clamped semiconductor optical amplifier (GCSOA) is presented. Both a timedomain and a small-signal analysis of those rate equations are used to investigate the crosstalk between different signal channels. It is shown that the crosstalk of GCSOA's strongly depends on the bit rate of the amplified signals and is lower at both very high bit rates and low bit rates. This crosstalk is proportional with the input power and, approximately, with the amplification.
\end{abstract}

Index Terms - Crosstalk, semiconductor optical amplifiers, small-signal analysis, time-domain analysis.

\section{INTRODUCTION}

A SEMICONDUCTOR optical amplifier (SOA) that is linear over a large input power range is a most attractive component for providing cost effective upgrades that will increase considerably the transmission capacity of a longdistance telecommunications network. This is particularly true for switching applications in WDM based optical networks [1], [2], where SOA's could provide very high on-off switching ratios as well as loss compensation. Recent progress in SOA technology has solved many of the problems (e.g., polarization sensitivity [3], [4] and low saturation output power [5]) which have prevented their widespread deployment in optical networks. However, a severe limitation for the use of SOA's in WDM applications is still the large crosstalk [6] between channels caused by gain saturation. A solution to this crosstalk problem is the gain clamped SOA (GCSOA), which has a fairly power independent gain as long as the amplified signal power is not much higher than the lasing power and thus results in a smaller crosstalk.

The efficiency of the gain clamping concept has already been demonstrated. Suppression of signal induced gain fluctuations using gain clamping by laser oscillation at a wavelength sufficiently different from the signal band has already been demonstrated in the case of a semiconductor amplifier with an external wavelength selective reflector [7] and also with integrated reflectors [8]. An in-depth theoretical analysis of the component has however not yet been reported.

To study the behavior of a GCSOA, we have developed a computer model based on rate equations. In this model,

Manuscript received June 10, 1997; revised October 16, 1997. This work has been performed in the framework of the European ACTS project OPEN and supported by the GOA-project "Broadband optical and mobile access networks" of the University of Gent, Belgium.

The authors are with the University of Gent-IMEC, Department of Information Technology, B-9000 Gent, Belgium.

Publisher Item Identifier S 1077-260X(97)09178-8.

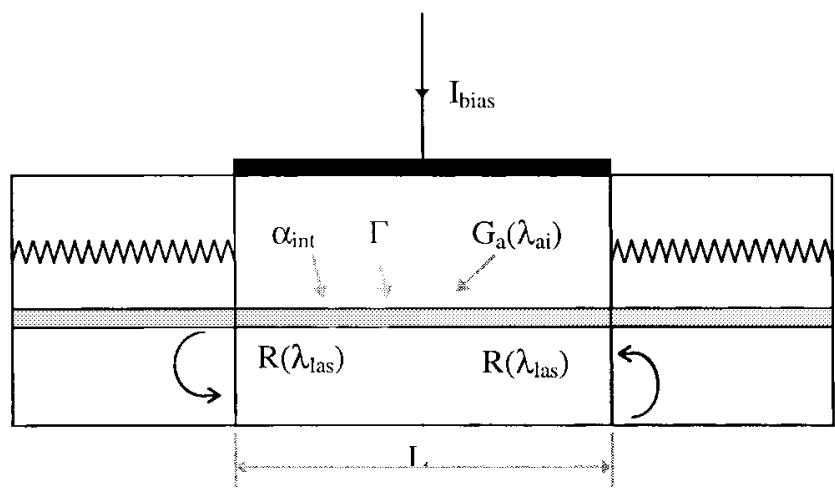

Fig. 1. Schematic structure of a GCSOA amplifier.

a separate photon density or power is used for each wavelength channel and as result four-wave mixing (FWM) is not included. We have used this model to investigate the AMcrosstalk (which is a result of the carrier depletion caused by the input signals) when different channels from a WDM system are injected into the amplifier. The channel spacing in a WDM system is typically 200 or $400 \mathrm{GHz}$ and FWM can be neglected in this case.

In Section II, the details of our time-domain GCSOA model, which is derived from the longitudinal equations and which includes spatial hole burning effects, and the numerical results obtained with this model will be given. In Section III, some analytical results obtained from a small-signal analysis will be provided. We show that by increasing the bit rate up to a certain level, one can decrease the crosstalk. In Section IV, some discussions of the influence of bias and device parameters on the performance of GCSOA's are made.

\section{TIME Domain Model OF GCSOA}

\section{A. The Rate Equation Model}

The axial structure of a GCSOA is shown in Fig. 1. In our analysis, we substitute the Bragg reflectors by discrete, wavelength dependent facet reflections with a reflectivity different from zero at the lasing wavelength $\lambda_{\text {las }}$ (with $\lambda_{\text {las }}=$ $1520 \mathrm{~nm}$ ) and zero reflectivity at the signal wavelengths $\lambda_{a i}$, where we assumed that the signal wavelengths are sufficiently far apart from the lasing wavelength.

The traveling-wave amplifier can be analyzed by assuming two different fields: the laser field of which we assume that it has a uniform photon density $S_{l}$ (because of a relatively 
large facet reflection and small gain coefficient) and the signal fields. We assume signal fields in four different channels that are all at wavelengths close to but a little larger than the laser's gain peak $\left(\lambda_{\max }=1550 \mathrm{~nm}\right)$ and with almost the same gain coefficient.

To simplify the analysis, we assume that only the dominant lasing mode is present in the GCSOA under investigation. We used the following rate equation model for the GCSOA. With $S_{l}$ and $S_{a i}$ the photon densities of the laser field and the signal fields respectively and $N$ the carrier density in the active layer, the rate equations are as follows [9]:

$$
\begin{aligned}
& \frac{d N}{d t}=\frac{I}{q V}-\frac{N}{\tau_{n}}-G_{l} S_{l}-G_{a} \sum_{i=1}^{m} S_{a i}(z, t) \\
& \frac{d S_{l}}{d t}=\left\{\frac{1}{L} \int_{0}^{L} G_{l}(z) d z-\gamma_{\text {as }}\right\} S_{l}+\frac{1}{L} \int_{0}^{L} \beta B N^{2} d z
\end{aligned}
$$

where " $m$ " indicates the total number of channels, $I$ is the bias current, $\tau_{n}$ represents the nonlinear carrier lifetime and $N / \tau_{n}$ is the spontaneous recombination rate, expressed as

$$
\frac{N}{\tau_{n}}=A N+B N^{2}+C N^{3}
$$

and $\beta$ is the fraction of the spontaneous emission that couples into the mode and $B$ is the bimolecular recombination coefficient. $S_{l}$ and $S_{a i}$ are effective photon densities, defined as the photon numbers per unit length divided by the cross section area of the active layer. Furthermore, we express $S_{a i}$ as

$$
S_{a i}(z, t)=S_{a i}(0, t) \exp \left\{\frac{1}{v_{g}} \int_{0}^{z}\left[G_{a}-\alpha_{\mathrm{int}} v_{g}\right] d z^{\prime}\right\} .
$$

The gain including nonlinear gain saturation is expressed as

$$
\begin{aligned}
G_{l, a}(z, t)= & G_{l, a}^{\prime}(z, t)\left[N(z, t)-N_{t}\right] \\
& \times\left[1-\varepsilon_{0,1} S_{l}(z, t)-\varepsilon_{1,0} S_{\text {in }}(z, t)\right]
\end{aligned}
$$

with

$$
\begin{aligned}
S_{\mathrm{in}} & =\sum_{i=1}^{m} S_{a i} \\
G_{l, a}^{\prime} & =\frac{d G_{l, a}}{d N}=v_{g} \Gamma \frac{d g_{l, a}}{d N} .
\end{aligned}
$$

$S_{\text {in }}$ is the total input photon density, $\Gamma$ is the confinement factor and $\varepsilon_{0}$ and $\varepsilon_{1}$ are the coefficients for self- and crossnonlinear gain suppression, respectively. We have assumed that $\varepsilon_{0}>\varepsilon_{1}$ and also that the differential gain coefficient $\frac{d g_{l, a}}{d N}$ is larger at the signal wavelengths $\lambda_{a i}$ than it is at the lasing wavelength $\lambda_{\text {las }}$, which means $\frac{d g_{a}}{d N}>\frac{d g_{l}}{d N}$. In (2), $\gamma_{\text {las }}$ is a constant which expresses the total loss in the laser cavity and through the mirrors. The unexplained symbols have their usual meaning.

We expand the carrier density $N(z, t)$ along the cavity direction $z$ as follows:

$$
N(z, t) \cong N_{0}(t)+N_{1}(t) f(z)
$$

where we have assumed $N_{1} \ll N_{0}$ and we have defined $f(z)$ as

$$
\begin{aligned}
f(z)= & \exp \left[\left(G_{0}-\alpha_{\mathrm{int}} v_{g}\right) \frac{z}{v_{g}}\right] \\
& -\frac{\exp \left[\left(G_{0}-\alpha_{\mathrm{int}} v_{g}\right) \frac{L}{v_{g}}\right]-1}{\left(G_{0}-\alpha_{\mathrm{int}} v_{g}\right) \frac{L}{v_{g}}}
\end{aligned}
$$

with

$$
\int_{0}^{L} f(z) d z=0
$$

and

$$
G_{0}=\frac{1}{L} \int_{0}^{L} G_{a}(z) d z
$$

By this definition, $N_{0}(t)$ and $G_{0}$ have the meaning of the average carrier density and signal gain along $z$, respectively, while the term $N_{1}(t) f(z)$ denotes the spatial deviations from the average $N_{0}(t) . N_{1}(t)$ will usually be negative, giving a decrease of $N(z, t)$ with $z$.

Hence, taking into account nonlinear gain suppression, $G_{l}$ should be

$$
\begin{aligned}
G_{l}=\left[G_{l}^{\prime}\right. & \left.\left(N_{0}+N_{1} f-N_{t}\right)\right] \\
& \times\left\{1-\varepsilon_{0} S_{l}-\varepsilon_{1} S_{\mathrm{in}}(0, t)\right. \\
& \times \exp \left[\left(G_{a}^{\prime}\left(N_{0}-N_{t}\right)-\alpha_{\mathrm{int}} v_{g}\right) \frac{z}{v_{g}}\right] \\
& \left.\times\left[1+\Gamma \frac{d g_{a}}{d N} N_{1} \int_{0}^{z} f d z\right]\right\}
\end{aligned}
$$

and the signal amplification is

$$
\begin{aligned}
A_{\text {sig }} & =\frac{S_{a i}(L, t)}{S_{a i}(0, t)} \\
& =\exp \left\{\frac{1}{v_{g}} \int_{0}^{L}\left(G_{a}^{\prime}\left[N(z, t)-N_{t}\right]-\alpha_{\text {int }} v_{g}\right) d z\right\} \\
& =\exp \left\{\left(G_{a}^{\prime}\left[N_{0}(t)-N_{t}\right]-\alpha_{\text {int }} v_{g}\right) \frac{L}{v_{g}}\right\}
\end{aligned}
$$

We have to substitute for $N$ in the carrier rate equations, with $S_{\mathrm{ino}}=S_{\mathrm{in}}(0, t)$ :

$$
\begin{aligned}
& \frac{d\left(N_{0}+N_{1} f\right)}{d t} \\
& =\frac{I}{q V}-\frac{N_{0}+N_{1} f}{\tau_{n}}-\left[G_{l}^{\prime}\left(N_{0}+N_{1} f-N_{t}\right)\right] \\
& \times\left\{1-\varepsilon_{0} S_{l}-\varepsilon_{1} S_{\mathrm{in} 0} \exp \left[\left(G_{a}^{\prime}\left[N_{0}-N_{t}\right]-\alpha_{\mathrm{int}} v_{g}\right) \frac{z}{v_{g}}\right]\right. \\
& \left.\times\left[1+\Gamma \frac{d g_{a}}{d N} N_{1} \int_{0}^{z} f d z\right]\right\} S_{l}-\left[G_{a}^{\prime}\left(N_{0}+N_{1} f-N_{t}\right)\right] \\
& \quad \times S_{\mathrm{in} 0} \exp \left\{\left[G_{a}^{\prime}\left(N_{0}-N_{t}\right)-\alpha_{\mathrm{int}} v_{g}\right] \frac{z}{v_{g}}\right\} \\
& \quad \times\left[1+\Gamma \frac{d g_{a}}{d N} N_{1} \int_{0}^{z} f d z\right] .
\end{aligned}
$$


From (14), one can derive the following equations for $N_{0}$ and $N_{1}$ :

$$
\begin{aligned}
\frac{d N_{0}}{d t}= & \frac{I}{q V}-\frac{N_{0}}{\tau_{n}}-G_{l}^{\prime}\left(N_{0}-N_{t}\right) \\
& \times\left[1-\varepsilon_{0} S_{l}-\varepsilon_{1} S_{\mathrm{in0}}\left(F_{00}+\Gamma \frac{d g_{a}}{d N} N_{1} F_{11}\right)\right] S_{l} \\
& +G_{l}^{\prime} N_{1} \varepsilon_{1} S_{\mathrm{in0}}\left(F_{10}+\Gamma \frac{d g_{a}}{d N} N_{1} F_{21}\right) S_{l} \\
& -G_{a}^{\prime}\left(N_{0}-N_{t}\right) S_{\mathrm{ino}}\left(F_{00}+\Gamma \frac{d g_{a}}{d N} N_{1} F_{11}\right) \\
& -G_{a}^{\prime} N_{1} S_{\mathrm{in0}}\left(F_{10}+\Gamma \frac{d g_{a}}{d N} N_{1} F_{21}\right) \\
\frac{d N_{1}}{d t}= & -\frac{N_{1}}{\tau_{n}}+G_{l}^{\prime}\left(N_{0}-N_{t}\right) \varepsilon_{1} S_{\mathrm{in0}}\left(\frac{F_{10}}{F_{0}}+\Gamma \frac{d g_{a}}{d N} N_{1} \frac{F_{21}}{F_{0}}\right) S_{l} \\
& -G_{l}^{\prime} N_{1}\left[1-\varepsilon_{0} S_{l}-\varepsilon_{1} S_{\mathrm{in0}}\left(\frac{F_{20}}{F_{0}}+\Gamma \frac{d g_{a}}{d N} N_{1} \frac{F_{31}}{F_{0}}\right)\right] S_{l} \\
& -G_{a}^{\prime}\left(N_{0}-N_{t}\right) S_{\mathrm{in0}}\left(\frac{F_{10}}{F_{0}}+\Gamma \frac{d g_{a}}{d N} N_{1} \frac{F_{21}}{F_{0}}\right) \\
& -G_{a}^{\prime} N_{1} S_{\mathrm{in0}}\left(\frac{F_{20}}{F_{0}}+\Gamma \frac{d g_{a}}{d N} N_{1} \frac{F_{31}}{F_{0}}\right) .
\end{aligned}
$$

For the laser's photon density equation:

$$
\begin{aligned}
\frac{d S_{l}}{d t}= & \left\{G _ { l } ^ { \prime } ( N _ { 0 } - N _ { t } ) \left[1-\varepsilon_{0} S_{l}-\varepsilon_{1} S_{\mathrm{in} 0}\right.\right. \\
& \left.\left.\times\left(F_{00}+\Gamma \frac{d g_{a}}{d N} N_{1} F_{11}\right)\right]\right\} S_{l} \\
& -\left\{G_{l}^{\prime} N_{1} \varepsilon_{1} S_{\mathrm{in} 0}\left[F_{10}+\Gamma \frac{d g_{a}}{d N} N_{1} F_{21}\right]+\gamma_{\mathrm{las}}\right\} \\
& \times S_{l}+\beta B\left(N_{0}^{2}+F_{0} N_{1}^{2}\right)
\end{aligned}
$$

where $F_{0}, F_{i j}$ are the coefficients defined as

$$
\begin{aligned}
F_{0}= & \frac{1}{L} \int_{0}^{L} f^{2} d z \\
F_{i 0}= & \frac{1}{L} \int_{0}^{L} \exp \left\{\left(G_{a}^{\prime}\left[N_{0}(t)-N_{t}\right]-\alpha_{\mathrm{int}} v_{g}\right) \frac{z}{v_{g}}\right\} f^{i} d z \\
F_{i 1}= & \frac{1}{L} \int_{0}^{L} \exp \left\{\left(G_{a}^{\prime}\left[N_{0}(t)-N_{t}\right]-\alpha_{\mathrm{int}} v_{g}\right) \frac{z}{v_{g}}\right\} \\
& \times f^{i-1} \int_{0}^{z} f\left(z^{\prime}\right) d z^{\prime} d z \quad i=0,1,2,3 .
\end{aligned}
$$

Equations (15)-(17) are our time-domain rate equations, which form the basis of our numerical model. In this model, the rate equations have been numerically solved by using a fifth-order Runge-Kutta method.

\section{B. Numerical Results}

The parameters we used in our simulation are shown in Table I.

For the input signals, we have chosen regular rectangular pulses and we define the signal gain as the output power divided by the input power, both taken in the middle of the bit period.

1) The Saturation Power: The saturation power is probably the most important parameter describing the behavior of
TABLE I

Parameters Used in the Simulations

\begin{tabular}{llll}
\hline Parameter & Symbol & Value & \\
\hline Length of GCSOA & $\mathrm{L}$ & 400 & $\mu \mathrm{m}$ \\
Stripe width & $\mathrm{w}$ & 1.5 & $\mu \mathrm{m}$ \\
Active layer thickness & $\mathrm{d}$ & 0.1 & $\mu \mathrm{m}$ \\
Effective group index of refraction in cavity & $\mathrm{n}_{\mathrm{g}}$ & 3.2 & \\
Lasing wavelength & $\lambda_{\mathrm{las}}$ & 1.52 & $\mu \mathrm{m}$ \\
Center signal wavelength & $\lambda$ & 1.558 & $\mu \mathrm{m}$ \\
Transparency carrier density & $\mathrm{N}_{\mathrm{t}}$ & $1.510^{6}$ & $\mu \mathrm{m}^{-3}$ \\
Differential gain coefficient of laser & $\mathrm{dg}_{\mathrm{I}}$ & $2.510^{-8}$ & $\mu \mathrm{m}^{2}$ \\
& $\mathrm{dN}$ & & \\
Differential gain coefficient of signal & $\mathrm{dg}$ & $9.510^{-8}$ & $\mu \mathrm{m}^{2}$ \\
\cline { 2 - 4 } & $\mathrm{dN}$ & & \\
Power confinement factor & $\Gamma$ & 0.2 & \\
Internal waveguide losses & $\alpha_{\mathrm{int}}$ & $2.010^{-3}$ & $\mu \mathrm{m}^{-1}$ \\
Linear recombination cocfficient & $\mathrm{A}$ & $510^{7}$ & $\mathrm{~s}^{-1}$ \\
Bimolecular recombination coefficient & $\mathrm{B}$ & 100 & $\mu \mathrm{m}^{3} / \mathrm{s}$ \\
Auger recombination coefficient & $\mathrm{C}$ & $7.510^{-5}$ & $\mu \mathrm{m}^{6} / \mathrm{s}$ \\
Spontaneous emission factor & $\beta$ & $210^{-5}$ & \\
Nonlinear self gain suppression coefficient & $\varepsilon_{0}$ & $310^{-6}$ & $\mu \mathrm{m}^{3}$ \\
Nonlinear cross gain suppression coefficient & $\varepsilon_{\mathrm{l}}$ & $210^{-6}$ & $\mu \mathrm{m}^{3}$ \\
\hline
\end{tabular}

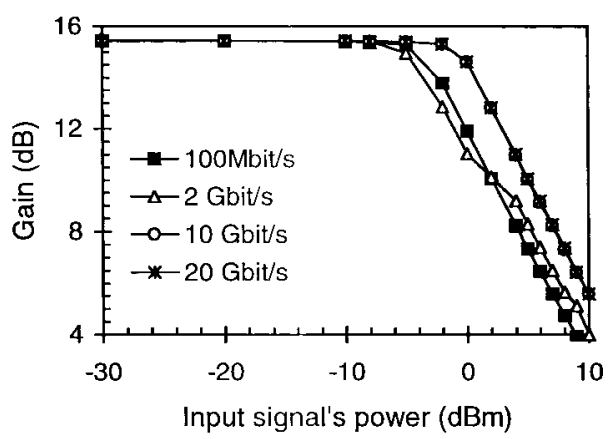

Fig. 2. Gain versus signal's input power with the bias current equals $40 \mathrm{~mA}$.

optical amplifiers in WDM systems, because an acceptably low crosstalk can only be obtained below this power level. Normally the saturation of the GCSOA starts at relatively higher power levels but occurs within a smaller power interval than in normal SOA's [10]. In our time-domain model, the saturation power is derived from the amplification versus input power. Fig. 2, showing the output power versus the input power for different bit rates and for a bias current of $40 \mathrm{~mA}$, illustrates that the saturation power is also depending on the bit rate. At very low bit rates, the saturation power is $3 \mathrm{~dB}$ lower than at high bit rates and even smaller saturation powers are found at certain intermediate bit rates (e.g., for $2 \mathrm{~Gb} / \mathrm{s}$ in Fig. 2). The reason for the high-saturation power at high bit rates is that at these very high bit rates, almost no modulation of the power in the lasing mode is obtained even if the signal power is modulated. The electron density can no longer follow the signal power variations. As a consequence, saturation at high bit rates is determined by the static average of the signal power, while at low bit rates it is determined by the peak value of the signal power that is twice the static average.

2) The Crosstalk: Because SOA's have the ability to amplify different signal channels simultaneously, the crosstalk between every channel in GCSOA's is the other property of concern. This crosstalk is mainly due to gain suppression and carrier density variations caused by the gain suppression. For simplicity, we took only two WDM channels to analyze the crosstalk and obtained from our time-domain model the 


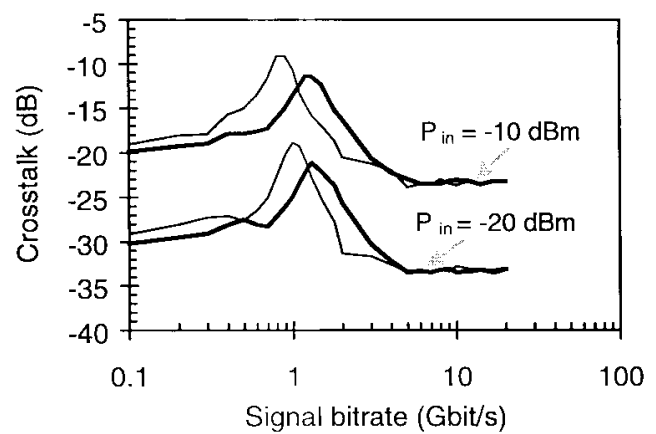

Fig. 3. Crosstalk versus signal bit rate for input powers of $-10 \mathrm{dBm}$ and $-20 \mathrm{dBm}$ at a bias current $I=40 \mathrm{~mA}$ (thin line), $60 \mathrm{~mA}$ (thick line).

results shown in Fig. 3. The crosstalk here has been defined as the change in average power during one bit period caused by the presence of another channel. The cross saturation characteristics between the two signal channels reveal that the degree of the gain saturation is uniquely determined by the total output power from both channels. There is also a resonance in the crosstalk, which obviously corresponds with the relaxation oscillation of the laser field.

\section{SMALl-Signal ANALYSIS}

\section{A. Approximations}

To derive analytical small-signal solutions, we first simplify the rate equations by neglecting the spatial variations. We are mainly interested in the crosstalk for input power levels that are significantly below the saturation power. The lasing mode is sufficiently above threshold in this case and therefore the spontaneous emission coupling into this mode can also be ignored. With $S_{l}$ and $S_{a}$ now the average photon densities of the laser field and the amplifier field, respectively, and $N$ the average carrier density in the active layer, the equations are

$$
\begin{aligned}
\frac{d N}{d t} & =\frac{\Delta I}{q V}-G_{l} S_{l}-G_{a} S_{a} \\
\frac{d S_{l}}{d t} & =\left[G_{l}-\gamma_{\text {las }}\right] S_{l}
\end{aligned}
$$

with

$$
G_{l, a}=G_{l, a}^{\prime}\left(N-N_{t}\right)\left[1-\varepsilon_{0,1} S_{l}-\varepsilon_{1,0} S_{a}\right]
$$

and with $\Delta I$ the difference between bias and threshold current.

It can be noticed that the spatial carrier density variations (which we assume to be mainly due to the spatial variation in the power of the amplified signal) could be included in the rate equations by modifying the stimulated emission term $G_{a} S_{a}$ in (21) to a term $G_{a} S_{a}\left(1-\varepsilon_{\text {spat }} S_{a}\right)$, with $\varepsilon_{\text {spat }}$ being time or frequency dependent and zero for high frequencies. One can easily proof this by substituting an approximate solution $N_{1}$ of (16) into the (15), a technique which has been used before in laser modeling [11]. We will discuss the effect of this extra gain suppression on our results later on.

As input signal, we use pulses of the form $S_{a}(t)=S_{a 0}[1+$ $\cos (\Omega t)]$, with $\Omega / \pi$ being the bit rate. We have assumed only one signal channel, hence the crosstalk is calculated as the influence of the signal on its own amplification.

We can derive small-signal AM responses using the usual harmonic formulation and approximating the variables in the rate equations as follows:

$$
\begin{aligned}
S_{a} & =S_{a 0}+\operatorname{Re}\left[S_{a 1} \exp (j \Omega t)\right] \\
S_{l} & =S_{l 0}+\operatorname{Re}\left[S_{l 1} \exp (j \Omega t)\right] \\
N & =N_{0}+\operatorname{Re}\left[N_{1} \exp (j \Omega t)\right] .
\end{aligned}
$$

The expansion for $S_{a}$ of course implies that $S_{a 1}=S_{a 0}$. In the static regime, we have

$$
\frac{\Delta I}{q V}=G_{l} S_{l 0}+G_{a} S_{a 0}
$$

which implies

$$
S_{l 0}=\frac{1}{G_{l}}\left[\frac{\Delta I}{q V}-G_{a} S_{a 0}\right]=\frac{1}{G_{l}^{\prime}}\left[\frac{G_{l}^{\prime}}{G_{l}} \frac{\Delta I}{q V}-G_{a}^{\prime} S_{a 0}\right] .
$$

The last expression in (28) is obtained under the approximation $G_{a} / G_{l}=G_{a}^{\prime} / G_{l}^{\prime}$ (which is equivalent to neglecting the gain suppression). Putting (24)-(26) and (28) into (21) and (22), we obtain

$$
\begin{aligned}
S_{l 1} & \cong \frac{S_{l 0}}{j \Omega+\varepsilon_{0} G_{l} S_{l 0}}\left[G_{l}^{\prime} N_{1}-\varepsilon_{1} G_{l} S_{a 0}\right] \\
{[j \Omega} & \left.+\frac{1}{\tau_{d}}+\frac{G_{l}^{\prime}}{G_{l}} \frac{\Delta I}{q V}\right] N_{1} \\
& \cong-G_{l} S_{l 1}-G_{a} S_{a 0}+\left(\varepsilon_{0} G_{l} S_{l 0}+\varepsilon_{l} G_{a} S_{a 0}\right) S_{l 1} \\
& +\left(\varepsilon_{1} G_{l} S_{l 0}+\varepsilon_{0} G_{a} S_{a 0}\right) S_{a 0}
\end{aligned}
$$

with $\tau_{d}$ the differential carrier lifetime. From (29) and (30), we can derive the expression for $S_{l 1} / S_{l 0}$, which is

$$
\frac{S_{l 1}}{S_{l 0}} \cong \frac{\left[\varepsilon_{1} G_{l}\left(j \Omega+\frac{1}{\tau_{d}}\right)+G_{a} G_{l}^{\prime}+\left(\varepsilon_{1}-\varepsilon_{0}\right) G_{a} G_{l}^{\prime} S_{a 0}\right] S_{a 0}}{j \Omega\left(\varepsilon_{0} G_{l} S_{l 0}+\frac{1}{\tau_{d}}+\frac{G_{l}^{\prime}}{G_{l}} \frac{\Delta I}{q^{V}}\right)+\left(G_{l} G_{l}^{\prime} S_{l 0}-\Omega^{2}\right)} .
$$

\section{B. The Crosstalk}

Using the small signal analysis, we can also derive simple expressions for the crosstalk at both high and low bit rates. The amplification $A_{a}$ of a signal in a WDM channel is given by

$$
A_{a}=\exp \left[G_{a}\left(N, S_{l}, S_{a i}\right) \frac{L}{v_{g}}-\alpha_{\text {int }} L\right] \text {. }
$$

This amplification can vary because of the fluctuation of the average carrier density, of the power in the laser field and of the signal power. The crosstalk in the output signal is therefore given by

$$
\frac{\Delta P_{\text {out }}}{P_{\text {out }}}=\frac{\Delta A_{a}}{A_{a}}=\Delta G_{a} \frac{L}{v_{g}} .
$$

From (29) and (30), one finds

$$
\begin{aligned}
\Delta G_{a} \cong & G_{a}^{\prime} N_{1}-\varepsilon_{0} G_{a} S_{a 0}-\varepsilon_{1} G_{a} S_{l 1} \\
\cong & \frac{G_{a}^{\prime}}{G_{l}^{\prime}} \frac{\left(j \Omega+\varepsilon_{0} S_{l 0} G_{l}\right) S_{l 1}+\varepsilon_{1} S_{l 0} G_{l} S_{a 0}}{S_{l 0}} \\
& -\varepsilon_{0} G_{a} S_{a 0}-\varepsilon_{1} G_{a} S_{l 1} \\
\cong & {\left[j \Omega \frac{G_{a}}{G_{l}}-\left(\varepsilon_{1}-\varepsilon_{0}\right) G_{a} S_{l 0}\right] \frac{S_{l 0}}{S_{l 0}} } \\
& +\left(\varepsilon_{1}-\varepsilon_{0}\right) G_{a} S_{a 0} .
\end{aligned}
$$

According to (31), at high bit rates (e.g., $\Omega>10 \mathrm{GHz}$ ):

$$
\frac{S_{l 1}}{S_{l 0}} \cong j \frac{\varepsilon_{1} G_{l}}{\Omega} S_{a 0}
$$




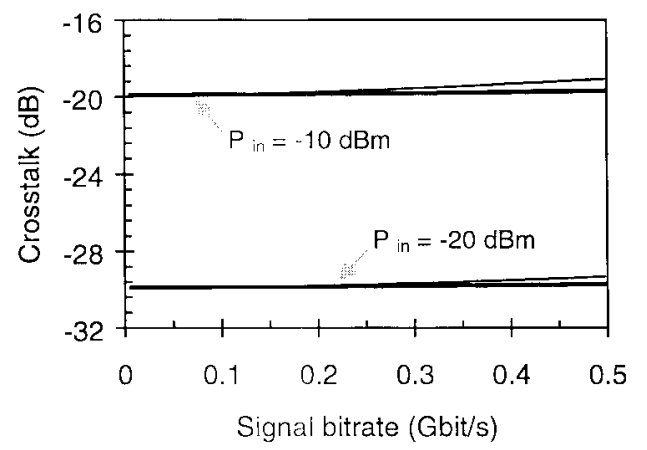

Fig. 4. Crosstalk versus signal bit rate at low bit rates for input powers of $-10 \mathrm{dBm}$ and $-20 \mathrm{dBm}$ at a bias current $I=40 \mathrm{~mA}$ (thin line), $60 \mathrm{~mA}$ (thick line).

and

$$
\Delta G_{a} \cong-\varepsilon_{0} G_{a} S_{a 0} .
$$

In (36), $S_{a 0}$ represents the average of the photon density, i.e.,

$$
\begin{aligned}
S_{a 0} & =\frac{1}{L} \int_{0}^{L} S_{a i}(z) d z=\frac{1}{L \hbar \omega_{a i} \mathrm{dw} v_{g}} \int_{0}^{L} P(z) d z \\
& =\frac{P_{\mathrm{in}, i}}{\hbar \omega_{a i} V} \frac{\exp \left[\left(G_{a}-\alpha_{\mathrm{int}} v_{g}\right) \frac{L}{v_{g}}\right]-1}{\left(G_{a}-\alpha_{\mathrm{int}} v_{g}\right)}
\end{aligned}
$$

where $\omega_{a i}$ is the angular frequency of channel $i$. Hence, the crosstalk at high bit rates is

$$
\frac{\Delta P_{\text {out }}}{P_{\text {out }}}=\frac{\Delta A_{a}}{A_{a}} \cong-\varepsilon_{0} G_{a} \frac{P_{\text {in }, i}}{\hbar \omega_{a i} V} \frac{A_{a}-1}{\ln \left(A_{a}\right)}\left(\frac{L}{v_{g}}\right)^{2} .
$$

From this expression, we can conclude that the crosstalk at high bit rates will no longer depend on the bias current and the bit rate, and it will only depend on the input signal's power and the gain suppression coefficient. Assuming $G_{a}$ to be considerably larger than $\alpha_{\text {int }}$, we can approximate $G_{a} L / v_{g}$ by $\ln \left(A_{a}\right)$ and we then find

$$
\frac{\Delta P_{\text {out }}}{P_{\text {out }}} \cong-\varepsilon_{0} \frac{P_{\text {in }, i}}{\hbar \omega_{a i} \text { w } d v_{g}}\left(A_{a}-1\right)
$$

By using (39) and our numerical parameters (Table I), we can predict a crosstalk at high bit rates of $-23 \mathrm{~dB}$ for an input power of $-10 \mathrm{dBm}$ and of $-33 \mathrm{~dB}$ for an input power of $-20 \mathrm{dBm}$. This is almost the same as we obtained from our time-domain model (Fig. 3).

From (31), one can also derive the expression for the crosstalk at lower bit rates:

$$
\begin{gathered}
\frac{S_{l 1}}{S_{l 0}} \cong-\frac{\left[G_{a} G_{l}^{\prime}+\left(\varepsilon_{1}-\varepsilon_{0}\right) G_{a} G_{l}^{\prime} S_{a 0}\right] S_{a 0}}{G_{l} G_{l}^{\prime} S_{l 0}} \cong-\frac{G_{a} S_{a 0}}{G_{l} S_{l 0}} \\
\Delta G_{a} \cong\left(\varepsilon_{1}-\varepsilon_{0}\right)\left(1+\frac{G_{a}}{G_{l}}\right) G_{a} S_{a 0}-j \Omega\left(\frac{G_{a}}{G_{l}} \frac{G_{a}^{\prime}}{G_{l}^{\prime}}\right) \frac{S_{a 0}}{S_{l 0}} .
\end{gathered}
$$

Hence, the crosstalk at low bit rates is

$$
\begin{aligned}
\frac{\Delta P_{\text {out }}}{P_{\text {out }}}=\frac{\Delta A_{a}}{A_{a}} \cong[ & \left(\varepsilon_{1}-\varepsilon_{0}\right)\left(1+\frac{G_{a}}{G_{l}}\right) G_{a} S_{a 0} \\
& \left.-j \Omega\left(\frac{G_{a}}{G_{l}} \frac{G_{a}^{\prime}}{G_{l}^{\prime}}\right) \frac{S_{a 0}}{S_{l 0}}\right]\left(\frac{L}{v_{g}}\right)
\end{aligned}
$$

in which $S_{l 0}$ also depends on $S_{a 0}$ according to (28).

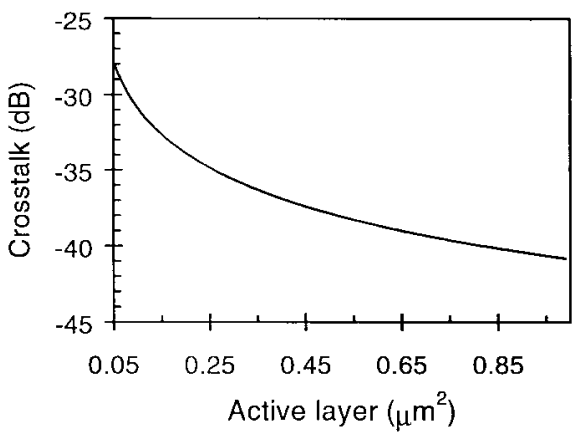

Fig. 5. The crosstalk versus SOA active layer's area at input power $=-20$ $\mathrm{dBm}$ and gain $=15.4 \mathrm{~dB}$.

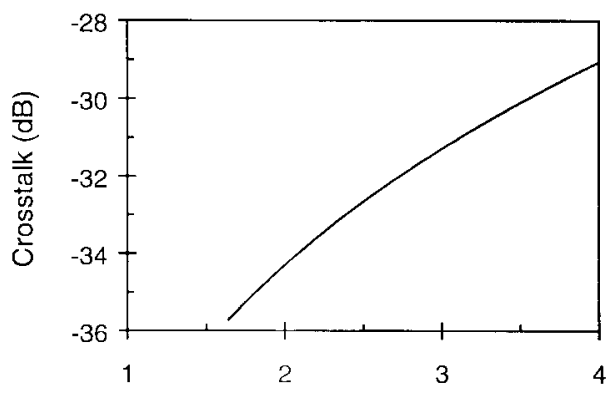

Ratio of differential gain coefficient $G_{a}{ }^{1} G_{\mid}^{\prime}$

Fig. 6. The crosstalk versus the ratio of the differential gain coefficient with input power $=20 \mathrm{dBm}$ at low bit-rate level.

The crosstalk at low bit rates obtained using the parameters of Table I is given in Fig. 4 for input power levels of -10 and $-20 \mathrm{dBm}$. These analytical results again agree very well with the results obtained using our time-domain model (Fig. 3). It can furthermore be noticed that if $\varepsilon_{1}$ is considerably smaller than $\varepsilon_{0}$ (as is often the case) and if $G_{a}$ is considerably larger than $G_{l}$, the value given by (42) will be $3 \mathrm{~dB}$ or more higher than the value given by (39). Hence, a smaller crosstalk results at high bit rates.

It can be noticed that the result for high bit rates is unaffected by the spatial carrier density variations since $\varepsilon_{\text {spat }}$ is nearly zero in this case. The carrier density can't follow the fast power fluctuations at these bit rates. However, spatial variations also have little influence at low bit rates. In the approximation for $S_{l 1} / S_{l 0}$ given in (40), gain suppression can be neglected and this also holds for the gain suppression caused by spatial hole burning. The variations in photon densities are determined by a balance in the total stimulated emission rate on which gain suppression has little effect. Spatial variations therefore only have an influence on the crosstalk near the resonance frequency of the relaxation oscillations.

\section{The Influence of DeVice Parameters}

In this section, we will investigate the influence of some device parameters on the crosstalk in more detail.

The crosstalk is strongly dependent on the waveguide dimensions $\mathrm{w}$ and $d$, as can be seen from (39). This dependence is illustrated in Fig. 5 and implies that a low crosstalk requires large active layer dimensions. 
The crosstalk at low bit rates strongly depends on the ratio of the differential gain coefficients $G_{a}^{\prime}$ and $G_{l}^{\prime}$. This ratio can be changed without changing the amplification by simultaneously also changing the internal loss and the facet reflectivity. This has been done to obtain the change of the crosstalk in Fig. 6 without change in amplification.

For the best performance of GCSOA, one can use shallow gratings (such that both $G_{l}$ and $G_{a}$ are large but $G_{a} / G_{l}$ is small) and keep the active layer's area large so as to reduce the crosstalk. Meanwhile, one can raise the bias current $\left[I-I_{\mathrm{th}}\right]$ for obtaining large saturation power.

\section{CONCLUSION}

A rate equation model for a GCSOA has been presented. This model has been used to investigate the crosstalk when several WDM channels are injected into the GCSOA. Using time-domain calculations as well as analytical small-signal solutions, we predicted theoretically that the crosstalk will be more than $3 \mathrm{~dB}$ lower at high bit rates than it is at low bit rates. In other words, by increasing the bit rate up to a certain level, one can decrease the crosstalk. Our analysis and conclusions are valid only for a regular variation between 0's and 1's. This can of course always be achieved with appropriate coding.

We have also discussed the dependence of crosstalk on device and bias parameters. The crosstalk at high bit rates strongly depends on the signal amplification, the input power and the waveguide dimensions. It is also proportional with the gain suppression coefficient, but is only weakly dependent on cavity length and is independent of the bias current. To obtain a small crosstalk, one can use shallow gratings and a large active layer area. A small amplification thus seems desirable to obtain a small crosstalk.

GCSOA's are attractive devices for amplifying a number of multiplexed channels simultaneously and it is feasible to integrate them with other devices on the same substrate. The analysis of GCSOA's given here is useful for its application in the future optical WDM systems [12].

\section{REFERENCES}

[1] T. Saitoh and T. Mukai, "1.5 $\mu \mathrm{m}$ GaInAsP traveling-wave semiconductor laser amplifier," IEEE J. Quantum Electron., vol. QE-23, pp. 1010-1020, June 1987

[2] P. Doussiere, A. Jourdan, G. Soulage, P. Garabedian, C. Graver, T. Fillion, D. Derouin, and D. Leclerc, "Clamped gain travelling wave semiconductor optical amplifier for wavelength division multiplexing applications," in 14th IEEE Int. Semiconduct. Laser Conf., Maui, HI, Sept. 1994, pp. 185-186.

[3] L. F. Tiemeijer, P. J. A. Thijs, T. van Dongen, R. W. M. Slootweg, J. M. M. van der Heijden, J. J. M. Binsma, and M. P. C. M. Krijn, "Polarization insensitive multiple quantum well laser amplifiers for the 1300 nm window," Appl. Phys. Lett., vol. 62, pp. 826-828, Feb. 1993.

[4] Ch. Holtmann, P.-A. Besse, and H. Melchior, IEEE Photon. Technol. Lett., vol. 8, pp. 343-345, Mar. 1996.

[5] T. Kamijoh, H. Horikawa, Y. Matsui, Y. K. Sin, M. Nakajima, C. Q. Xu, and Y. Ogawa, "Improved operation characteristics of long-wavelength lasers using strained MQW active layers," IEEE J. Quantum Electron., vol. 30, pp. 524-532, Feb. 1994.

[6] H. E. Lassen, P. B. Hansen, and K. E. Stubkjaer, "Crosstalk in 1.5$\mu \mathrm{m}$ InGaAsP optical amplifiers," J. Lightwave Technol., vol. 6, pp. $1559-1565$, Oct. 1988

[7] J. C. Simon, P. Doussiere, P. Lamouler, I. Valiente, and F. Riou, "Travelling wave semiconductor optical amplifier with reduced nonlinear distortions," Electron Lett., vol. 30, no. 1, pp. 49-50, Jan. 1994.
[8] L. F. Tiemeijer, P. J. A. Thijs, T. v. Dongen, J. J. M. Binsma, E. J. Jansen, and H. R. J. R. van Helleputte, "Reduced intermodulation distortion in $1300 \mathrm{~nm}$ gain-clamped MQW laser amplifiers," IEEE Photon. Technol. Lett., vol. 7, pp. 284-286, Mar. 1995.

[9] J. Kinoshita, "Modeling of high-speed DFB lasers considering the spatial holeburning effect using three rate equations," IEEE J. Quantum Electron., vol. 30, pp. 929-938, Apr. 1994.

[10] B. Bauer, F. Henry, and R. Schimpe, "Gain stabilization of a semiconductor optical amplifier by distributed feedback," IEEE Photon. Technol. Lett., vol. 6, pp. 182-185, Feb. 1994.

[11] R. H. Wentworth, "Large-scale longitudinal spatial-hole-burning contribution to laser gain compression," IEEE J. Quantum Electron., vol. 29, pp. 2145-2153, July 1993.

[12] M. J. O'Mahony, "Semiconductor optical amplifier for use in future fiber systems," J. Lightwave Technol., vol. 6, pp. 531-544, Apr. 1988.

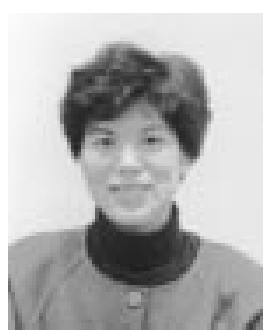

Jinying Sun was born in Jiangsu, China. She received the B.Sc. degree in physics from the University of Suzhou, China, in 1983, and the M.Sc. degree in solid state physics from the University of Innermongolia, China, in 1987, respectively. She is currently a Ph.D. candidate in applied physics in the University of Gent, Gent, Belgium.

Since 1987, she joined in the East-China Shipbuilding Institute and Jiangsu Synthesize Technical Institute, P.R. China, as a researcher. Since 1996, she has been with the Department of the Information Technology (INTEC) of the University of Gent as a Research Assistant. She has worked in the field of analysis and characterization of WDM-component for high bit rate optical communication system.

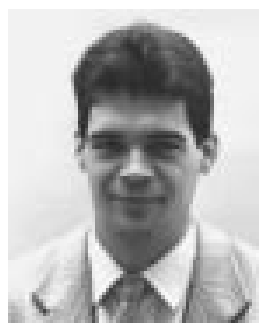

Geert Morthier (M'93) was born in Gent, Belgium, on March 20, 1964. He received the degree in electrical engineering and the Ph.D. dgree from the University of Gent in 1987, 1991, resepectively.

Since 1991, he is a member of the permanent staff of IMEC. His main interests are in the modeling and characterization of optoelectronic components. He has authored or coauthored over 50 papers in the field. He is also one of the two authors of the Handbook of Distributed Feedback Laser (Artech House, 1997).

Dr. Morthier is a member of the Optical Society of America and the Flemish Engineers Association.

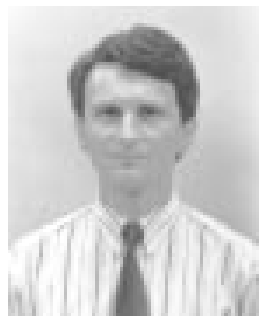

Roel Baets (M'88) received the degree in electrical engineering from the University of Gent, Gent, Belgium, in 1980, the M.Sc. degree in electrical engineering from Stanford University, Stanford, CA, in 1981, and the Ph.D. degree from the University of Gent, in 1984.

Since 1981, he has been with the Department of Information Technology (INTEC) of the University of Gent. Since 1989, he has been a Professor in the Engineering Faculty of the University of Gent. From 1990 to 1994, he has also been a part-time Professor at the Technical University of Delft, Delft, The Netherlands. He has worked in the field of III-V devices for optoelectronic systems. With over 100 publications and conference papers, he has made contributions to the modeling of semiconductor laser diodes, passive guided wave devices and to the design and fabrication of OEIC's. His main interests now are the modeling, design and testing of optoelectronic devices, circuits and systems for optical communication and optical interconnect.

Dr. Baets is a member of Optical Society of the America, IEEE Lasers and Opto-Electronics Society, SPIE, and the Flemish Engineer Association. He has been member of the program committees of OFC, ECOC, IEEE Semiconductor Laser Conference, ESSDERC, CLEO-Europe, and the European Conference on Integrated optics. 